ORI GI NAL

\title{
Caracterización fisiológica del periodo posparto en cuatro grupos raciales bovinos en el trópico colombiano
}

\section{Physiological characterization of the postpartum period in four cattle breeds in the tropics of Colombia}

\author{
Henry Grajales L, ${ }^{1 *}$ Ph.D, Aureliano Hernández V, ${ }^{1}$ Ph.D, Esperanza Prieto, ${ }^{2}$ M.Sc.
}

\begin{abstract}
${ }^{1}$ Universidad Nacional de Colombia, Facultad de Medicina Veterinaria y de Zootecnia Departamento de Ciencias para la Producción Animal, Departamento de Ciencias para la Producción Animal, Grupo de investigación en Fisiología de la Reproducción. Bogotá, CoIombia. ${ }^{2}$ Corporación Colombiana de Investigación Agropecuaria - CORPOICA. Turipana, Cereté, Colombia.* Correspondencia: hagrajalesl@unal.edu.co
\end{abstract}

Recibido: Febrero 13 de 2009; Aceptado: Octubre 20 de 2009.

\section{RESUMEN}

Objetivo. Caracterizar el periodo posparto a través de los parámetros reproductivos, los niveles de progesterona y los tipos de actividad ovárica. Materiales y métodos. 63 hembras de los grupos raciales Holstein x Cebú (HC), Simmental x Cebú (SC), Romosinuano (R) y Cebú (C) se siguieron desde el destete hasta confirmación de segunda gestación, se tomaron muestras de sangre para determinar las concentraciones de progesterona mediante Radioinmunoanálisis. Para el análisis de la información se utilizó el programa estadístico SAS. Resultados. Se presentaron diferencias significativas para la edad al primer calor posparto, excepto en los grupos SC y HC; respecto al peso al primer calor posparto, los grupos SC y C presentaron mayores tasas de crecimiento, seguidos por los grupos R y HC. No se encontraron diferencias significativas para el intervalo parto primer incremento de progesterona. La primera ovulación posparto se presentó hacia el día 34.45 en el grupo SC, 83.24 en el HC, 46.13 en el R y 31.9 en el C. El tipo de actividad ovárica posparto refleja el desempeño en la eficiencia reproductiva, los grupo SC y HC que presentaron bajas tasas de concepción, contrario a el grupo $\mathrm{R}$, que presentó una excelente tasa de concepción y niveles de progesterona considerados normales en más del $80 \%$ de las hembras. Conclusiones. Los perfiles de progesterona ofrecen un método conveniente para monitorear la actividad ovárica, pudiendo determinar con precisión la duración de la aciclicidad, el momento de la reactivación ovárica, presentación de disfunciones ováricas y el inicio y mantenimiento de la preñez.

Palabras clave: Actividad ovárica, genotipo, posparto, progesterona, trópico. 


\section{ABSTRACT}

Objective. To describe the postpartum period through reproductive parameters, progesterone levels and types of ovarian activity. Materials and methods. 63 females of the breed Holstein x Cebu (HC), Simmental x Cebu (SC), Romosinuano (R) and Cebu (C) were followed from weaning to confirmation of a second pregnancy, blood samples were taken to determine progesterone concentrations by Radioimmunoassay. For the analysis of the information statistical program SAS was used. Results. There were significant differences for age at first postpartum heat, except in the $\mathrm{SC}$ and $\mathrm{HC}$ groups, regarding the first heat postpartum weight, SC and $\mathrm{C}$ groups had higher growth rates, followed by $\mathrm{R}$ and $\mathrm{HC}$ groups. No significant differences were found for the interval from calving to first progesterone rise. The first postpartum ovulation was presented to the 34.45 days in the SC group, 83.24 in the HC, 46.13 in the R and 31.9 in C. The type of ovarian activity reflects the reproductive efficiency performance, the SC and HC groups had low conception rates, contrary to the R group, which presented an excellent conception rates and progesterone levels considered normal in more than $80 \%$ females. Conclusions. The analysis of the progesterone profiles offer a convenient method for monitoring ovarian activity, being able to accurately determined the acyclic duration, the time of ovarian activity resumption, presence of ovarian dysfunctions and finally the beginning and pregnancy maintenance.

Key words: Ovarian activity, genotype, postpartum, progesterone, tropic.

\section{NTRODUCCIÓN}

El ganado bovino en su naturaleza es poliéstrico, pero experimenta un periodo de infertilidad posparto que varia en su duración y es dependiente de múltiples factores. Este periodo de quiescencia reproductiva se compone de un periodo inicial de anovulación (falta de ciclos ovulatorios recurrentes) y anestro (ausencia de la expresión del estro) (1). El anestro posparto, puede ser definido como la ausencia de conducta estral y ovulación acompañada de concentraciones séricas menores a $0.2 \mathrm{ng} / \mathrm{ml}$ (2). La duración del anestro posparto es regulada principalmente por dos factores: el amamantamiento y el consumo de nutrientes antes y después del parto (3). El amamantamiento interfiere con la liberación hipotalámica de la hormona liberadora de gonadotropinas $(\mathrm{G} n \mathrm{RH})$, cuya cantidad resulta insuficiente para estimular a la hipófisis, provocando una marcada supresión en la liberación pulsátil de la hormona luteinizante (LH). Tal inhibición de la liberación episódica de LH se da probablemente mediante la secreción de un péptido opiode hipotalámico $\beta$-endorfina, en respuesta al estímulo del amamantamiento (4).
La complejidad de la relación entre el estado nutricional y la función reproductiva ha sido señalada y ampliamente revisada. Se ha sugerido que la concentración de glucosa sanguínea es el factor que vincula el estado nutricional con la función $\beta$ reproductiva a nivel del hipotálamo. Vacas con concentraciones de glucosa reducidas, disminuyen su cantidad de progesterona en plasma. Por otro lado, las reservas corporales de grasa regulan la secreción de hormonas hipotalámicas e hipofisiarias, el déficit de energía posparto, perjudica la actividad reproductiva mediante la supresión de la liberación de GnRH y por tanto de la frecuencia pulsátil de LH, necesaria para el crecimiento folicular (4). También se ha relacionado el intervalo posparto con los cambios en las hormonas metabólicas, estas hormonas (Factor de crecimiento similar a la insulina (IGF-I), insulina y leptina) pueden influenciar la secreción de $\mathrm{GnRH}$, actuando sobre las neuronas que contienen $\mathrm{GnRH}$, en las vías neuronales que afectan a estas neuronas, o en los gonadotrofos ubicados en la hipófisis. Así mismo estas pueden también actuar directamente en el ovario 
influenciando su sensibilidad a las gonadotropinas (5). El anestro posparto también es afectado por varios factores "menores", entre estos se incluyen la estación, raza, edad, número de partos, presencia del toro y efectos de arrastre de preñeces previas. (6). En muchos trabajos se ha observado que el genotipo tiene un efecto significativo en la reproducción posparto en ganado tropical; se plantea que el ganado proveniente de zonas templadas y sus cruces, presentan menores intervalos entre partos comparadas con razas cebuinas (7).

El reinicio de la actividad ovárica posparto, se da como consecuencia de un incremento en las concentraciones de la hormona folículo estimulante (FSH), 3 a 5 días posparto, encontrándose que la primera onda de desarrollo folicular, puede producir un folículo dominante hacia el día 7-10 posparto, sin embargo, el desarrollo final de los folículos y la capacidad de ovular, se obtienen gracias a la recuperación de la secreción pulsátil de Ia LH (8). La actividad del ovario es generalmente reflejada por la presencia o ausencia de un cuerpo lúteo, de allí que muchos investigadores hayan puesto especial atención a la posibilidad de las mediciones de progesterona para proveer una medida confiable de la presencia y actividad secretoria del tejido luteal (9).

Es importante mencionar que los prolongados periodos de anestro (mayores a 150 días), son característicos de vacas Bos indicus y Bos taurus/Bos indicus en regiones tropicales, siendo uno de los mayores problemas de infertilidad existentes, debido a su alta incidencia y a las pérdidas económicas que de el se derivan, pues constituye la mayor limitante para lograr el intervalo entre partos ideal de doce meses (10). Son importantes las investigaciones en condiciones de trópico cálido-húmedo acerca del comportamiento fisiológico posparto comparativo entre ganado cruzado de doble propósito, criollo y Cebú.

El objetivo de este trabajo fue caracterizar el periodo posparto a través de la edad y peso al primer calor posparto (EcPto, PcPto) edad y peso a la segunda concepción $\left(\mathrm{EC}_{2}, \mathrm{PC}_{2}\right)$, el intervalo parto primer incremento de progesterona $\left(\mathrm{IPP}_{4}\right)$, la descripción de los niveles de progesterona (NP) y los tipos de actividad ovárica $(A O)$ en hembras de los grupos raciales Holstein x Cebú (HC), Simmental x Cebú (SC), Romosinuano (R) y Cebú (C).

\section{MATERI ALES Y MÉTODOS}

Sitio de estudio. El trabajo se realizó en el centro de investigaciones Turipaná de la Corporación Colombiana para la Investigación Agropecuaria (CORPOICA), ubicado en el noroccidente colombiano (trópico cálidohúmedo) a $20 \mathrm{msnm}$, con una temperatura promedio de $27.5^{\circ} \mathrm{C}, 1200 \mathrm{~mm} /$ año de precipitación y una humedad relativa promedio del $83 \%$.Geográficamente se encuentra a $8^{\circ}$ y $5^{\prime}$ de latitud Norte y $75^{\circ}$ y $49^{\prime}$ de longitud occidente, con respecto al meridiano de Greenwich. Los animales fueron mantenidos en praderas mixtas compuestas por, Dichantium aristatum (Angleton) y, Brachiaria mutica (Pará), con acceso a sal mineralizada ( $8 \%$ P) y agua a voluntad; se realizaron controles periódicos para determinar la composición y disponibilidad de forraje, para mantener el cubrimiento de las necesidades nutricionales de los animales y procurar durante todo el periodo de seguimiento un comportamiento fisiológico normal.

Animales. Se escogieron al azar 63 hembras de primer parto, así: $11 \mathrm{~F}_{1}$ Simmental x Cebú $(\mathrm{SC}) ; 25 \mathrm{~F}_{1}$ Holstein $x$ Cebú $(\mathrm{HC}) ; 16$ Romosinuano (R) y 11 Cebú (C). A partir del parto se hizo seguimiento individual de los animales dos veces al día durante una hora, de 6 a 7 a.m. y de 5 a 6 p.m., para registrar la presencia de calores (una hembra se consideró en celo cuando se dejó montar dos o más veces por otras hembras o por el macho marcador o cuando montó a otras hembras); todos los animales fueron pesados cada quince días y al momento de presentar los celos.

\section{Obtención de muestras y} radioinmunoanálisis. Se tomaron muestras de sangre venosa, mediante la técnica de venopunción de la yugular, a 
partir del día 20 pos-parto, cada cinco días hasta la aparición del primer calor observado posparto, momento en el que se hizo el servicio por monta natural controlada con los toros asignados según el programa para control de consanguinidad dentro del hato. El mismo protocolo de muestreo se siguió cada cinco días pos servicio (tomando como día 0 el día del servicio) hasta la confirmación de preñez, que se hizo el día 35 pos-servicio; se tuvieron al menos tres calores servidos por hembra. Las muestras de sangre, se centrifugaron a $2500 \mathrm{rpm}$ por 15 minutos para separar el suero de los elementos formes; el suero se almacenó en viales y se mantuvo en congelación $\left(-20^{\circ} \mathrm{C}\right)$ hasta la cuantificación de los niveles de progesterona por la técnica del radioinmunoanálisis en fase sólida (RIA) $(11,12)$, para lo cual se utilizaron kits Coat-A-Count Progesterone de la Diagnostics Products Corporation (DPC), el cual presenta una sensibilidad de $0.02 \mathrm{ng} /$ $\mathrm{ml}$ y una reactividad cruzada muy baja con otros componentes presentes en la muestra. Las muestras fueron corridas en secuencias diferentes, obteniéndose un coeficiente de variación intra-ensayo de $8.79 \%$ e interensayo de $10.38 \%$.

Criterios para manejo de variables. El $\mathrm{IPP}_{4}$, se determinó en forma directa a partir del chequeo y análisis de los protocolos diseñados para el manejo de los datos y/o en forma indirecta con base en el chequeo y análisis de los perfiles de progesterona. El criterio base para la clasificación del tipo de actividad ovárica, de acuerdo a los perfiles de progesterona, fue el empleado por Opsomer $(9,13)$. Se consideró como Reactivación Ovárica posparto cuando se manifestó un incremento que excedió 1 ng/ $\mathrm{ml}$ en los niveles de progesterona, al menos en un muestreo, para definir ciclicidad regular se tomó el comportamiento estral, con o sin manifestación de calor observado, que estuvo respaldado por una función luteal basada en el mantenimiento de los niveles de progesterona por encima de $2 \mathrm{ng} / \mathrm{ml}$ presente por un periodo de tiempo determinado o de manera cíclica (14).

Análisis estadístico. Utilizando estadística descriptiva se observó y analizó el comportamiento de las variables descritas dentro y entre los grupos raciales, usando para el análisis el procedimiento GLM del programa de análisis estadístico SAS (15).Para comparar el comportamiento de los NP en el posparto temprano, entre los grupos raciales evaluados, se empleó la prueba de Duncan para la comparación de medias y análisis de varianza.

\section{RESULTADOS}

Edad y peso al primer calor posparto y a la segunda concepción. Se presentaron diferencias significativas para la EcPto entre todos los grupos, a excepción de los promedios de los grupos SC y HC; en cuanto al PcPto, los grupos SC y C presentan una mayor tasa de crecimiento, seguidos por el grupo R de comportamiento intermedio y el grupo HC que presenta un tamaño corporal pequeño (Tabla 1 ). En referencia a $E_{2}$ no hubo diferencias significativas $(p<0.001)$ entre los grupos SC y HC, los cuales tuvieron diferencias significativas en la $\mathrm{PC}_{2}$; entre los grupos $\mathrm{R}$ y $\mathrm{C}$ se presentaron diferencias significativas $(p>0.001)$ para la $\mathrm{EC}_{2}$ y la $\mathrm{PC}_{2}$ (Tabla 2$)$.

Tabla 1. Edad y peso al primer calor posparto en bovinos en el trópico cálido-húmedo colombiano.

\begin{tabular}{cccccc}
\hline \multicolumn{5}{c}{ Grupo Racial } \\
& SC (11) & HC (25) & R (16) & C (11) \\
\hline \multirow{2}{*}{ EcPto, dias } & 855.36 & 854.6 & 1051.9 & 1196.5 \\
& $\pm 38^{\mathrm{c}}$ & $\pm 82.7^{\circ}$ & $\pm 50.4^{\circ}$ & $\pm 61.6^{\mathrm{s}}$ \\
PcPto, kg & 447.91 & 390.4 & 426.6 & 452.7 \\
& $\pm 44.6^{\mathrm{a}}$ & $\pm 44.7^{\circ}$ & $\pm 31.9^{\mathrm{a}}$ & $\pm 49.2^{\mathrm{s}}$ \\
\hline
\end{tabular}

Promedios con la misma letra no son significativamente diferentes $(p<0.0001)$.

Tabla 2. Edad y peso a la segunda concepción en bovinos en el trópico cálido-húmedo colombiano.

\begin{tabular}{ccccc}
\hline \multicolumn{5}{c}{ Grupo Racial } \\
& SC (11) & HC (25) & R (16) & C (11) \\
\hline \multirow{2}{*}{$\mathrm{EC}_{21}$ dias } & 887.1 & 900.1 & 1040.5 & 1251.5 \\
& $\pm 75.4^{\mathrm{c}}$ & $\pm 12.2^{\mathrm{c}}$ & $\pm 73.5^{\mathrm{b}}$ & $\pm 11.1^{\mathrm{s}}$ \\
$\mathrm{PC}_{21} \mathrm{~kg}$ & 456.2 & 399.3 & 422.4 & 457.8 \\
& $\pm 38.8^{\circ}$ & $\pm 46.1^{\circ}$ & $\pm 33^{\circ}$ & $\pm 26.9^{\mathrm{s}}$ \\
\hline
\end{tabular}

Promedios con la misma letra no son significativamente diferentes $(p<0.0001)$. 
I ntervalo parto primer incremento de progesterona posparto. Aunque no se encuentran diferencias significativas entre grupos para el IPP ${ }_{4}$, es notable que los grupos $\mathrm{HC}$ y C, presentan un mayor intervalo de tiempo en la recuperación ovárica posparto, indicando el alto grado de heterogeneidad dentro de estos grupos raciales (Tabla 3).

Niveles de progesterona y actividad ovárica. El valor promedio de progesterona para el periodo de inactividad ovárica fue de $0.0607 \pm 0.496 \mathrm{ng} / \mathrm{ml}$ presentado por el grupo $\mathrm{R}, 0.0862 \pm 0.094 \mathrm{ng} / \mathrm{ml}$ para el grupo $\mathrm{HC}$ y $0.1080 \pm 0.099 \mathrm{ng} / \mathrm{ml}$ para el grupo SC. El

Tabla 3. Primer incremento de progesterona en el posparto para cada grupo racial evaluado.

\begin{tabular}{ccccc}
\hline \multicolumn{5}{c}{ Grupo Racial * } \\
& SC (11) & HC (25) & R (16) & C (11) \\
\hline IPP $_{4}$ & 39.45 & 88.24 & 51.13 & 36.9 \\
& \pm 24.12 & \pm 76.7 & \pm 23.26 & \pm 22.1 \\
\hline
\end{tabular}

* = Promedio + Desviación estándar tipo de AO posparto refleja el desempeño en la eficiencia reproductiva, en donde los grupo SC y HC tienen tasas de concepción muy bajas, contrario a lo que muestra el grupo $\mathrm{R}$, que presenta una excelente tasa de concepción y tiene NP considerados normales en más del $80 \%$ de las hembras (Tabla 4).

Tabla 4. Tipo de actividad ovárica posparto (\%) presentada por grupos raciales bovinos en el trópico cálido - húmedo colombiano.

Grupo Racial

Tipo de actividad ovárica SC HC R (11) (25) (16)

Tipo 1-a Normal alta

$\begin{array}{lll}27.2 & 12.5 & 25\end{array}$

Tipo 1-b Normal media

$\begin{array}{lll}18.2 & 12.5 & 31.3\end{array}$

Tipo 1-c Normal baja

$\begin{array}{lll}18.2 & 16.7 & 31.3\end{array}$

Tipo 2 Media

$\begin{array}{lll}18.2 & 16.7 & 0.0\end{array}$

$\begin{array}{llll}\text { Tipo } 3 \text { Cesación de la ciclicidad } & 18.2 & 16.7 & 12.4\end{array}$ \begin{tabular}{llll} 
Tipo 4 Anovulación prolongada & 0.0 & 24.9 & 0.0 \\
\hline
\end{tabular}

Por problemas técnicos no se obtuvo la información de la raza Cebú.
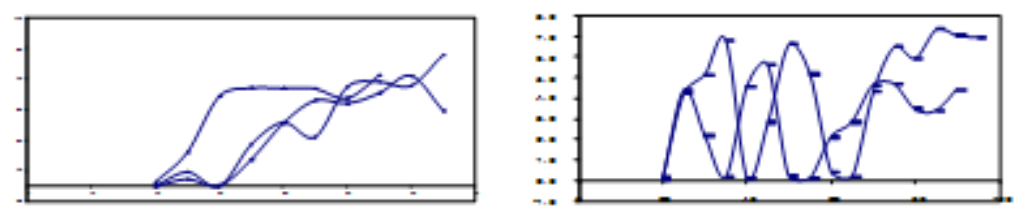

a) Tipo 1-a; $27.2 \%$ de las hembras

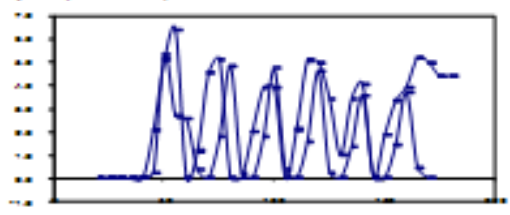

b) Tipo 1-b; $18.2 \%$ de las hembras

c) Tipo 1-c; $18.2 \%$ de las hembras
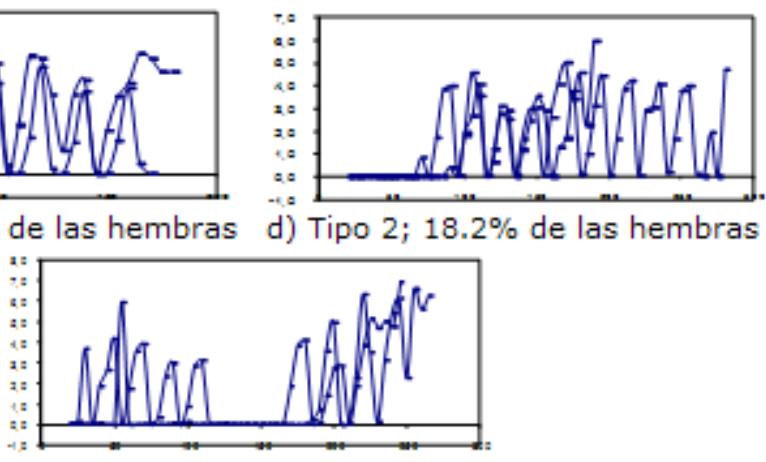

e) Tipo 3; $18.2 \%$ de las hembras

Figura 1. Tipos de actividad ovárica presentada por el grupo SC. (Eje de las X "Días posparto" eje de las $Y$ "P4(ng/ml)") 


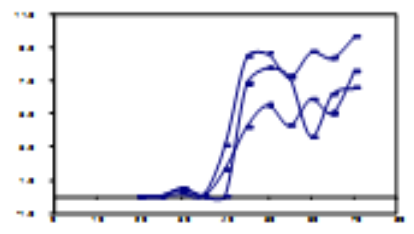

a) Tipo 1 -a; $12.5 \%$

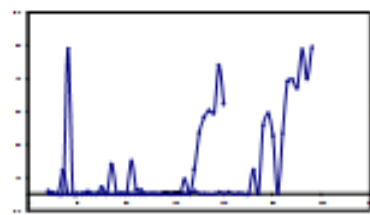

e) Tipo $3 ; 16.7 \%$

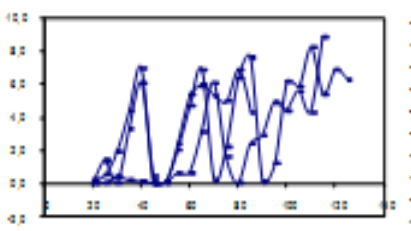

b) Tipo 1-b; $12.5 \%$

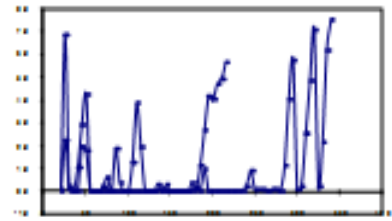

e) Tipo $3 ; 16.7 \%$

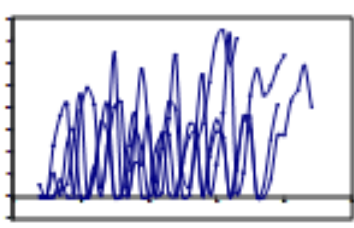

c) Tipo $1-$ c; $16.7 \%$

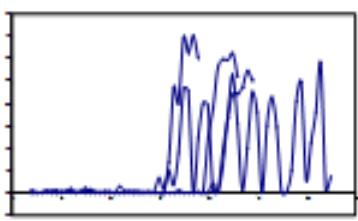

f) Tipo $4 ; 24.9 \%$

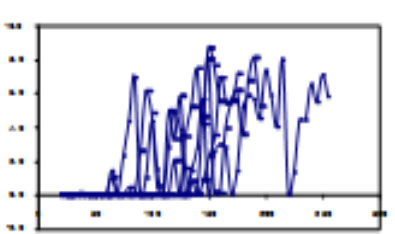

d) Tipo 2; $16.7 \%$

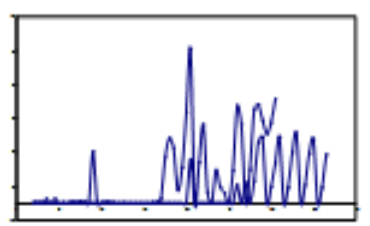

f) Tipo $4 ; 24.9 \%$

Figura 2. Tipos de actividad ovárica presentada por el grupo HC. (Eje de las X "Días posparto" eje de las $Y$ “P4(ng/ml)").

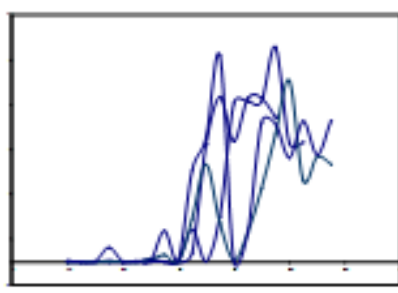

a) Tipo $1-a ; 25.0 \%$

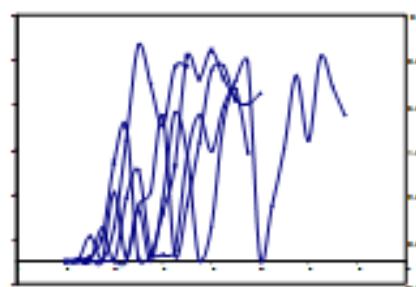

b) Tipo $1-b ; 31.3 \%$

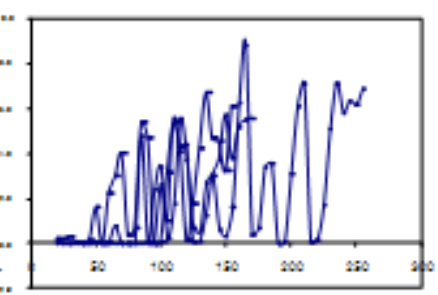

c) Tipo $1-\mathrm{c} ; 31.3 \%$

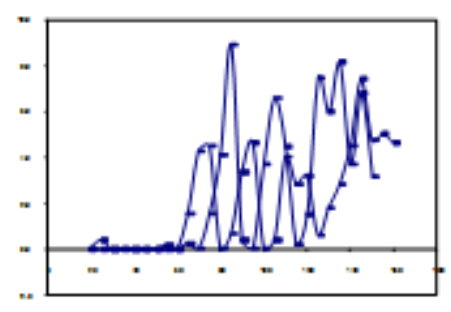

c) Tipo 1 -c; $31.3 \%$

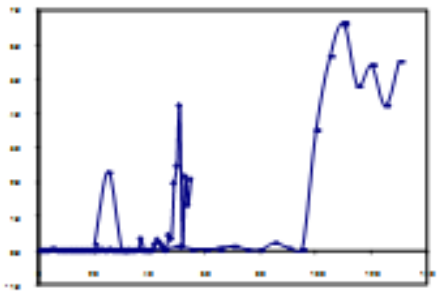

d) Tipo 3; $12.4 \%$

Figura 3. Tipo de actividad ovárica presentada por el grupo R. (Eje de las X "Días posparto" eje de las $Y$ "P4(ng/ml)").

Al examinar los resultados se puede observar (Figuras 1 a 3 ) como en el grupo SC se presentan un $36.4 \%$ de perfiles Tipo 2 y 3 , en el grupo $\mathrm{HC}$ se tienen un $58.3 \%$ de perfiles Tipo 2, 3 y 4, mientras en el grupo $\mathrm{R}$ tan solo se encuentran un $12.4 \%$ de perfiles Tipo 3; los tipos de actividad ovárica 2, 3 y 4 muestran un modelo que se puede considerar como de baja eficiencia reproductiva en el periodo posparto, siendo las más importantes disfunciones la cesación de la ciclicidad y la anovulación prolongada. Por otra parte, se observa como el grupo R presenta un $87.6 \%$ de perfiles Tipo 1 en comparación con un $63.6 \%$ del grupo SC y un $41.7 \%$ del grupo HC, que destaca el excelente comportamiento reproductivo de las hembras R.

La primera ovulación posparto se presenta hacia los 34.45 días en el grupo SC, 83,24 días en el HC, 46.13 días en el $\mathrm{R}$ y 31.9 días en el $\mathrm{C}$.

\section{SCUSIÓN}

En términos generales, el efecto benéfico positivo que determina la precocidad a la pubertad para el grupo HC, reportada por Grajales (16) en un estudio previo realizado 
con el mismo grupo animal, se pierde cuando se evalúa la EcPto y $E_{2}$, pues no se conservan las diferencias con el grupo SC, que eran evidentes a la edad a la pubertad y se disminuyen las que se observan al compararlo con los grupos R y C (16). Los menores valores encontrados para la EcPto en los grupos raciales HC y SC, comparados con las razas $\mathrm{R}$ y $\mathrm{C}$ pueden ser debidos a que en estos dos primeros genotipos se involucra una raza de tipo lechero, las cuales reinician su actividad ovárica más rápido que los bovinos productores de carne $(17,18)$. Asimismo las diferencias encontradas para la variable EcPto entre los genotipos R y C, puede deberse a que la especie Bos indicus presenta un anestro posparto más prolongado que la especie Bos taurus $(19,20)$.

Los grupos evaluados, y en particular el SC y el HC, mostraron una edad a la segunda concepción (determinada por la edad al primer parto) bastante inferior cuando se compara con los datos reportados para grupos raciales semejantes en la zona (38 a 41 meses para edad a primer parto, según Navarrete (21).

Para Opsomer (13) el primer incremento significativo de progesterona se presenta en promedio el día 37 posparto teniendo una desviación estándar de 26 días, lo cual permite apreciar que los resultados mostrados por los grupos SC y C coinciden estrechamente con los reportados; los resultados del grupo $\mathrm{R}$ permanecen dentro del rango descrito; $y$ los del grupo HC difieren ampliamente, mostrando una gran desviación estándar, lo cual ha sido un hecho coincidente en muchos de los trabajos reportados por otros investigadores $(9,22)$.

Se debe precisar que es bastante difícil el comparar los resultados obtenidos en este estudio con resultados previos de otros investigadores $(13,9)$ dada la gran variabilidad que se puede tener en la definición para determinar qué es normalidad o anormalidad.

Gunzler (23) y Bulman y Wood (24) reportan en sus trabajos la presencia de un 81.6 y $22.5 \%$, respectivamente de perfiles de progesterona definidos como anormales, estando los valores obtenidos para los grupos SC y HC dentro de este rango, mientras el del grupo R esta bastante por debajo del rango mínimo. El grupo R continua destacándose al compararlo con el reporte de Kassa (25), pues estos solo encontraron un $66 \%$ de vacas con perfil de progesterona normal. En un estudio realizado por Báez (26), se reporta en ganado Costeño con Cuernos, un genotipo criollo, que el $90 \%$ de las vacas presentó actividad normal (tipo 1) y el $10 \%$ actividad media (tipo 2 ).

Se presentan grandes diferencias entre los grupos evaluados, evidenciando que existen factores intrínsecos al grupo racial e incluso al individuo que pueden estar determinando las condiciones de la reactivación ovárica posparto y la presentación de desajustes hormonales o disturbios en la actividad ovárica.

En este trabajo se logró identificar: i). Que la primera ovulación posparto se presenta hacia los 34.45 días en el grupo SC, a los 83,24 días en el grupo HC, a los 46.13 días en el grupo $\mathrm{R}$ y a los 31.9 días en el grupo C; ii). Que el intervalo de recuperación posparto medido como el I $\mathrm{PP}_{4}$ puede servir como un indicador del inicio de la actividad ovárica que puede ayudar en la determinación precisa de la aciclicidad y en la evaluación de disfunciones ováricas; iii). Que el grupo SC presentó un 36.4\% de perfiles Tipo 2 y 3 , el grupo $\mathrm{HC}$ un $58.3 \%$ de perfiles Tipo 2, 3 y 4, mientras el grupo $R$ tan solo tuvo un $12.4 \%$ de perfiles Tipo 3 , que los tipos de actividad ovárica 2, 3 y 4 muestran un modelo que se puede considerar como de baja eficiencia reproductiva en el periodo posparto, siendo las más importantes disfunciones la cesación de la ciclicidad y la anovulación prolongada. El grupo R presenta un $87.6 \%$ de perfiles Tipo 1 en comparación con un $63.6 \%$ del grupo SC y un $41.7 \%$ del grupo HC, que destaca el excelente comportamiento reproductivo de las hembras $R$.

Asimismo se puede concluir que el análisis de los perfiles de progesterona ofrecen un método conveniente para el monitoreo de la actividad ovárica, pudiendo determinar con precisión la duración de la aciclicidad, 
el momento de la reactivación ovárica, la presentación de disfunciones ováricas y finalmente el inicio y mantenimiento de la preñez.

\section{Agradecimientos}

Los autores agradecen al apoyo financiero y logístico para la realización de esta investigación a COLCIENCIAS .

\section{REFERENCI AS}

1. Stevenson J S, Lamb GC, Hoffmann DP, Minton JE. Interrelationships of lactation and postpartum anovulation in suckled and milked cows. Livest Prod Sci 1997; 50:57-74.

2. Esperón A. Efecto de la transferencia de tecnología y su impacto en reproducción en el sistema de producción bovina de doble propósito en el oriente de Colima [Tesis doctoral]. Colima, México: Universidad de Colima.; 2000.

3. Wettemann RP, Lents CA, Ciccioli NH, White FJ, Rubio I. Nutritional- and sucklingmediated anovulation in beef cows. J Anim Sci 2003; 81:E48-59.

4. Ahuja C. Tasa de gestación en vacas anéstricas Bos taurus / Bos indicus después de la inducción a la ovulación usando GnRH más Prostaglandina F2á [Tesis de maestría]. Colima, México: Universidad de Colima.; 2003.

5. Lucy MC. Physiological mechanisms leading to reproductive decline in dairy cattle. En: Reproductive loss in dairy cows: Is the trend reversible?. St. Paul, Minnesota, USA; Proceedings of the II Bi-Annual W.E. Petersen Symposium: 2003.

6. Short RE, Bellows RA, Staigmiller RB, Berardinelli JG Custer EE. Physiological mechanisms controlling anestrus and infertility in postpartum beef cattle. J Anim Sci 1990; 68: 799-816.

7. Abeygunawardena $\mathrm{H}$, Dematawewa CMB. Pre-pubertal and postpartum anestrus in tropical Zebu cattle. Anim Reprod Sci 2004; 82-83: 373-387.
8. Crowe MA. Resumption of ovarian cyclicity in post-partum beef and dairy cows. Reprod Domest Anim 2008; 43 Supl 5: 20-28.

9. Opsomer G, Coryn M, Kruif A. Measurement of ovarian cyclicity in the postpartum dairy cow by progesterone analysis. Reprod Domest Anim 1999; 34: 297-300.

10. Montiel F, Ahuja C. Body condition and suckling as factors influencing the duration of postpartum anestrus in cattle: a review. Anim Reprod Sci 2005; 85: 1-26.

11. Prieto ME. Edad y peso al inicio de la pubertad y seguimiento de los tres primeros ciclos estrales en novillas Holstein [Tesis demaestría]. Bogotá, Colombia: Universidad Nacional de Colombia; 1993.

12. Cardozo CJ. Relación entre factores climáticos y algunas características del ciclo estral en novillas Holstein de la Sabana de Bogotá [tesis de maestría]. Bogotá, Colombia: Universidad Nacional de Colombia; 1993.

13. Opsomer G, Coryn M, Deluyker H, Kruif A. An analysis of ovarian dysfunction in high yielding dairy cows after calving, based on progesterone profiles. Reprod Domest Anim 1998; 33: 193-204.

14. Wehrman ME, Kojima FN, Sanchez T, Mariscal DV, Kinder JE. Incidence of precocious puberty in developing beef heifers. J Anim Sci 1996; 74: 2462-2467.

15. SAS/STAT: Guide for Personal Computer [programa de ordenador]. Versión 6.12. Cary (NC): SAS Institute Incorporation; 1998. 
16. Grajales H, Hernández A, Prieto E. Edad y peso a la pubertad y su relación con la eficiencia reproductiva de grupos raciales bovinos en el trópico colombiano. Livestock Research for Rural Development [en línea] 2006 Oct [citado 3 Dic 2008]; 18(139). URL Disponible en: http://www.Irrd.org/ Irrd18/10/graj 18139.htm

17. AntaJE. Análisis de la información publicada sobre la eficiencia reproductiva del ganado bovino en el trópico mexicano [Tesis de licenciatura]. Ciudad de México, México: Universidad Nacional Autónoma de México; 1987.

18. Córdova A, Pérez JF. Indicadores reproductivos de bovinos en el trópico mexicano y factores que lo determinan. Med Vet 2002; 19 (3): 47-56.

19. Castillo JH, Ruiz Cortés ZT, Olivera Ángel M, Jiménez C. Reactivación ovárica postparto en vacas cebú Brahman con relación al peso y condición corporal. Rev Col Cienc Pec 1997; 10: 12-18.

20. Montaño E, Ruiz Cortés ZT. ¿Por qué no ovulan los primeros folículos dominantes de las vacas cebú posparto en el trópico colombiano? Rev Col Cienc Pec 2005; 18 (2): 127-135.

21. Navarrete MS, Abuabara YP, Mendoza GP, Martínez GS, Corredor GS, Serrano GD et al. Evaluación de la reproducción en ganaderías de doble propósito en Córdoba. Avances en Monitoreo Ganadero. Proyecto Colombo-Alemán. 1995.
22. Mihm M. Delayed resumption of cyclicity in postpartum dairy and beef cows. Reprod Domest Anim 1999; 34: 276-284.

23. Gunzler O, Muller S, Claus R, Karg H, Pirchner F. Analyse von Fortpflanzungsproblemen bei Kuhen mit Hilfe der Progesteronbestimmung im Milchfett. Zuchthygiene 1982; 17: 193-202.

24. Bulman DC, Wood PDP. Abnormal patterns of ovarian activity in dairy cows and their relationships with reproductive performance. Anim Prod 1980; 30: 177-188.

25. Kassa T, Ahlin K-A, Larsson K. Profiles of progesterone in milk and clinical ovarian findings in post partum cows with ovarian dysfunctions. Nord Vet Med 1986; 38: 360-369.

26. Báez GM, Grajales H, Pérez JE. Caracterización del ciclo estral mediante perfiles de esteroides ( progesterona, 17âestradiol) en la raza Costeño con Cuernos (Bos taurus) en el trópico Colombiano. Livestock Research for Rural Development [en línea] 2007 Sept [citado 3 Dic 2008]; 19 (132). Disponible en: URL: http:// www.Irrd.org/Irrd19/9/baez19132.htm 